\section{Lithium encephalopathy}

\author{
D Smith MRCPI P Keane MB J Donovan MB ${ }^{1}$ \\ K Malone FRCPI $^{1} \quad T$ J McKenna FRCPI FRCP
}

J R Soc Med 2003;96:590-591

In a patient receiving lithium for psychiatric disorder, the onset of other disease and its treatment seems to have destabilized lithium control.

\section{CASE HISTORY}

A man aged 67 was admitted after an episode of transient left leg weakness, lasting 20 minutes. He was known to have type 2 diabetes, treated by diet but poorly controlled (glycosylated haemoglobin 11.8\% [normal 4.4-6.0]). There was a long history of bipolar affective disorder treated with lithium carbonate $400 \mathrm{mg}$ twice daily and more recently low-dose venlafaxine. On admission his serum lithium was $0.35 \mathrm{mmol} / \mathrm{L}$ (therapeutic range $0.4-1.1$ ). CT of the brain was normal. A transient ischaemic attack was diagnosed and he was discharged home on metformin, gliclazide, pravastatin, aspirin and Asasantin Retard (dipyridamole and aspirin) as well as his usual maintenance dose of lithium carbonate and venlafaxine.

Eight days later he returned, having experienced confusion, anorexia and generalized shaking for three days. Random blood glucose was $3.0 \mathrm{mmol} / \mathrm{L}$, blood urea $10.0 \mathrm{mmol} / \mathrm{L}$ (2.5-6.6), serum creatinine $154 \mu \mathrm{mol} / \mathrm{L}$ (62-115). He was judged to have symptomatic hypoglycaemia and was treated with oral Lucozade and a maintenance infusion of $5 \%$ dextrose. The hypoglycaemic medications were stopped. Next day his blood glucose concentrations were in the normal range but his symptoms were worse. He was increasingly drowsy, obtunded, and abulic and he had developed a coarse tremor with myoclonic jerks of his arms and legs. Repeat CT and MRI of the brain were normal. Serum and urine toxicology were negative. Cerebrospinal fluid white cell count was normal, protein $0.73 \mathrm{~g} / \mathrm{L}(0.15-0.45)$, glucose $6.4 \mathrm{mmol} / \mathrm{L}$ (blood glucose $8.5 \mathrm{mmol} / \mathrm{L})$. Serum lithium was raised at $2.02 \mathrm{mmol} / \mathrm{L}$. The electroencephalogram showed diffuse slowing throughout the brain with no epileptiform activity. Encephalopathy secondary to lithium intoxication was diagnosed and all his psychotropic medications were stopped. He was treated with isotonic saline as fluid

Departments of Endocrinology and ${ }^{1}$ Psychiatry, St Vincent's University Hospital, Elm Park, Dublin 4, Ireland

Correspondence to: Dr Diarmuid Smith, Department of Endocrinology, St Vincent's University Hospital, Elm Park, Dublin 4, Ireland replacement and with three sessions of haemodialysis with a blood flow of $170 \mathrm{~mL} / \mathrm{min}$. Lithium levels fell to undetectable. His tremor and myoclonic jerks stopped and over the following week his toxic encephalopathy resolved. Detailed psychiatric evaluation, and checks with his local pharmacy and general practitioner on the dose and quantity of his tablets, yielded no evidence of a deliberate or accidental overdose.

\section{COMMENT}

Lithium intoxication has various clinical manifestations but neurological symptoms predominate. ${ }^{1}$ The onset tends to be gradual, with initial confusion, then impaired consciousness leading to coma and occasionally death. ${ }^{2}$ Neurological effects include seizures, choreiform and parkinsonian movements, and cerebellar signs (dysarthria, ataxic gait, incoordination). ${ }^{1,2}$ The symptoms do not necessarily correlate with lithium concentrations: toxicity has been reported at therapeutic levels and negligible symptoms at high levels. ${ }^{3}$ As a general rule aggressive treatment (intravenous fluids, haemodialysis) should be given when serum lithium exceeds $2 \mathrm{mmol} / \mathrm{L}$, but this decision should be governed more by the clinical features than by the spot lithium level. ${ }^{3}$

The reason why in our patient the lithium concentration rose so rapidly from the subtherapeutic to the toxic range over a week is not clear. The medications prescribed after his first admission have not been reported to interfere with lithium and the combination of lithium and venlafaxine seems well tolerated in patients with bipolar affective disorder. Dehydration might have interfered with renal clearance and so raised the serum lithium, but the patient was clinically envolaemic. Oakley et al. ${ }^{4}$ have reported on 28 patients with severe lithium neurotoxicity, 26 of whom had chronic poisoning and the remaining 2 acute-on-chronic poisoning. They identified three risk factors contributing independently to lithium toxicity — namely, nephrogenic diabetes insipidus, age $>50$ years and thyroid dysfunction. Subnormal endogenous creatinine clearance was also implicated.

Haemodialysis is the cornerstone of treatment for lithium toxicity: with its low atomic weight and negligible protein binding, lithium is one of the most readily dialysable toxins. ${ }^{5}$ Haemodialysis should be stopped when the lithium concentration falls to the therapeutic range but may need to be repeated since rebound can occur, with high concentrations, after a treatment session.

\section{REFERENCES}

1 Ilagan MC, Carlson D, Madden JF. Lithium toxicity: 2 case reports. Del Med J 2002; 74:263-70 
2 Meltzer E, Steinlauf S. The clinical manifestations of lithium intoxication. Isr Med Assoc J 2002;4:265-7

3 Nagappan R, Parkin WG, Holdsworth SR. Acute lithium intoxication Anaesth Intensive Care 2002;30:90-2

4 Oakley PW, Whyte IM, Carter GL. Lithium toxicity: an iatrogenic problem in susceptible individuals. Aust N Z J Psychiatry 2001;35:833-40

5 Fenves AZ, Emmett M, White MG. Lithium intoxication associated with acute renal failure. South Med J 1984;77:1472-4

\section{Anticoagulation in a head-injured patient}

\author{
Ahmed Ibrahim MBBS MRCS \\ Keyoumars Ashkan MRCP FRCS \\ Martin Smith MBBS FRCA \\ Lawrence Watkins MA FRCS
}

J R Soc Med 2003;96:591-592

The use of low-molecular-weight heparin in neurosurgical patients is controversial.

\section{CASE HISTORY}

A man of 53 sustained a small frontal contusion in a fall (Figure 1). Initially, he was fully alert and oriented and he was admitted for observation to the local hospital. There was no relevant medical history, and blood results including a clotting screen were normal. A week later he became acutely breathless and was found to have a $\mathrm{PaO}_{2}$ of $9 \mathrm{kPa}$ and raised D-dimers. A pulmonary embolus was suspected and he was started on enoxaparin $120 \mathrm{mg}$ once a day. Within a few hours of receiving the first dose of this lowmolecular-weight heparin the patient's level of consciousness began to deteriorate. He was opening his eyes, making incomprehensible sounds and flexing his limbs to painful stimuli (Glasgow coma score 9). A repeat head scan revealed considerable progression of the frontal contusion (Figure 2). After intubation he was transferred to our unit, and on arrival his activated partial thromboplastin time (APTT) was 67 seconds (normal range 22-41). He was managed conservatively in the intensive care unit; an intracranial pressure monitor was not inserted for fear of inducing further haemorrhage. The next day spiral CT of the chest showed no evidence of pulmonary embolus. The

Departments of Neurosurgery and Neuroanaesthesia, National Hospital for Neurology and Neurosurgery, London, UK

Correspondence to: Dr Ahmed Ibrahim, 38 C Camden Road, London NW1 9DR, UK

E-mail: aibrahim@doctors.net.uk
APTT gradually improved and was normal within four days. Thereafter he made a good recovery.

\section{COMMENT}

In patients already receiving anticoagulants, the special hazards of head injury are well known. In a retrospective study the case fatality was 50\% compared with $20 \%$ in a group not receiving anticoagulants. ${ }^{1}$ The question of thromboembolism prophylaxis in neurosurgical patients is difficult: these patients are commonly immobile for long periods and are prone to deep vein thrombosis, yet haemorrhage into the operation site is more dangerous than in other forms of surgery where heparin prophylaxis is advocated. Nurmohamed and co-workers ${ }^{2}$ used fractionated or low-molecular-weight heparin in addition to compression stockings postoperatively in neurosurgical patients and reported a clinically significant decrease in venous thromboembolism without increased risk of major bleeding, and Constantini et al. ${ }^{3}$ found no excess risk from perioperative low-molecular-weight heparin, in a randomized comparison with $0.9 \%$ saline, in patients having brain surgery. These studies were in elective neurosurgery. In emergency work, Norwood and colleagues ${ }^{4}$ gave enoxaparin, $30 \mathrm{mg}$ 12-hourly, to 177 patients admitted with blunt injury and with documented intracranial haemorrhage. Progression of intracranial haemorrhage, on CT, was seen in $4 \%$ and the authors concluded that enoxaparin could

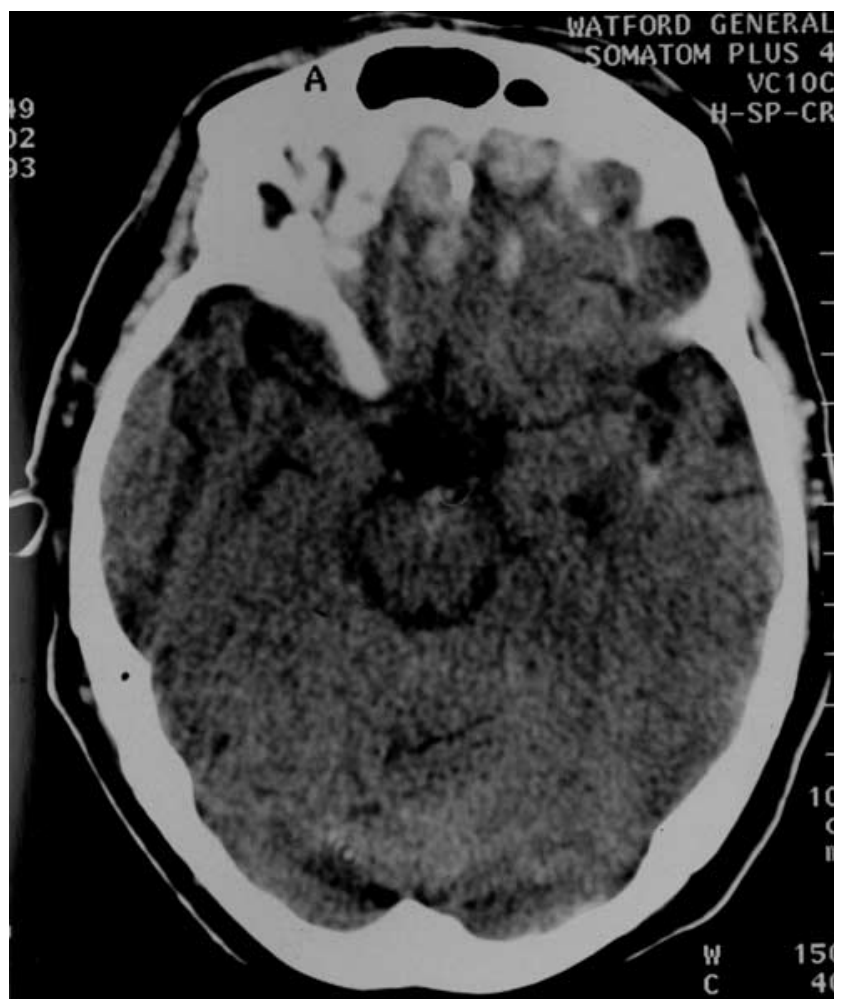

Figure $1 \mathbf{C T}$ brain scan showing bifrontal contusions 